SCRDSI-2021, Aravali Institute of Technical Studies, Udaipur (Rajasthan), India

International Journal of Technical Research \& Science (Special Issue) ISSN No.:2454-2024 (online)

\title{
LATEST TRENDS IN SOFTWARE ENGINEERING RESEARCH
}

\author{
Devendra Suthar \\ E-Mail Id: dev_arya123@gmail.com \\ Aravali Institute of Technical Studies, Udaipur, India
}

\begin{abstract}
Software engineering is dynamic trains that have persistent development in research in distinguishing new strategies, devices and approaches that have cause huge enhancement in programming advancement and support to be progressively dependable and proficient. Past research faultfinders on cost decrease, quality and adaptability have interminable attempt to plan and create numerous approaches to enhance these divisions are as yet making effects the product business. [1]The new patterns in software engineering exploration themes settle under the examination field of Cloud Computing, Big Data, Android Computing, Network Security and Software Engineering Project Management. Nevertheless, there are progressively other research territories in programming building that have been serious explored and executed in the ventures.
\end{abstract}

Keywords: Cloud Computing, Android Computing, Big Data, Network Security, Project Management.

\section{CLOUD COMPUTING}

Cloud computing a new research area in the field of software engineering where so many new methodologies and models are launched with sake to the industry and likewise furnish information with intension to enhance the training and programming industry in giving decrease cost and enhance the present innovation in the business [1]; [2]. Based from past research, issues in distinguishing the nature of administrations in cloud computing are fairly poor and as a result of assortment of administrations gave in cloud computing are disregarded, generally in administration segment where the advantage of cloud computing isn't felt by the product business and the clients. Thus researcher Abdelmaboud, et al. [2] Have acquainted five-inquire about centre zone with enhance distributed computing administrations. The examination zones are as in Figure 1 beneath.

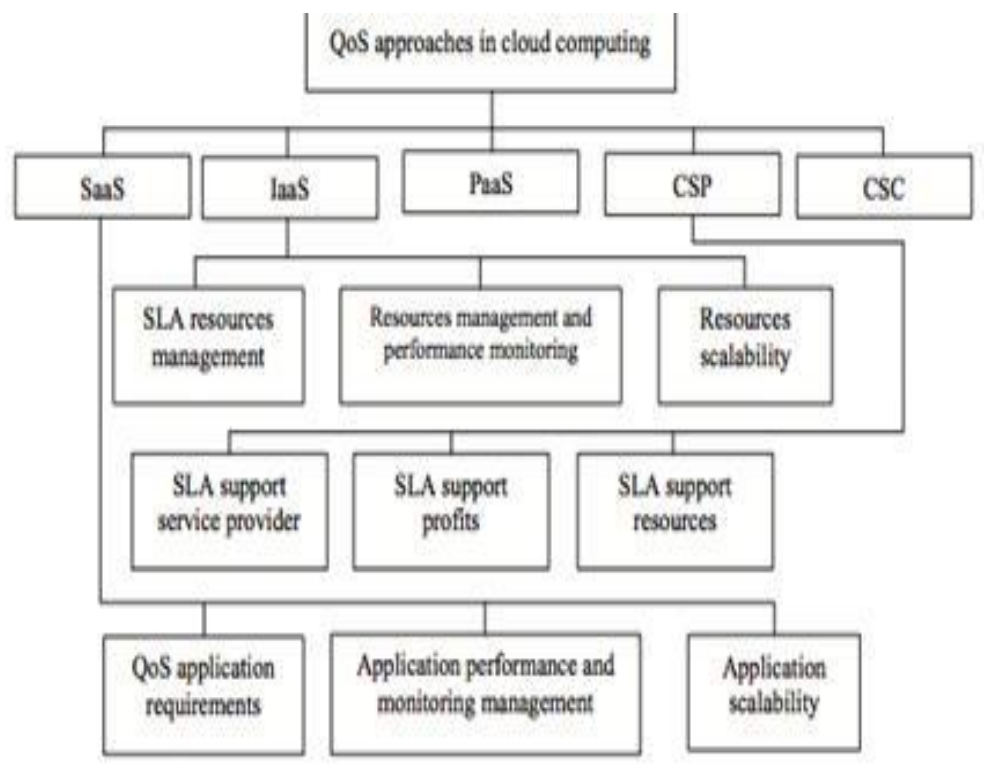

Fig. 1.1 The characteristics of quality of services in cloud computing

This SaaS research is associated with the applications given in systems that behave as service to shoppers. Paas analysis target development of the platform resource for applications and system services. Iaas analysis target information centres and virtualization resources in organizations. Whereas CSP focus to the suppliers of cloud computing services like computer code, computer code platform and infrastructure services to the users. Finally CSC associated with the individuals and therefore the organization United Nations agency use cloud computing services just like the computer code, platform etc. Another conference paper associated with cloud computing is that the adoption of cloud computing for software package engineering learning surroundings through Saas and amphibian genus [1] analysis study. Problems highlighted during this paper are associated with the supply, maintenance, accessibility, quantifiability, compatibility and resource utilization of software package and hardware tools utilized in software package engineering course. Another issue highlighted is that the disposition to adapt this new technology. From the collective review of past survey handled with 3 universities that are Asia Pacific University of Technology and Innovation Asian country, University Technology Asian country and University Asian nation Asian country, discussion of results obtained wherever software package engineering students face problem of compatibility, availableness and licensing of the software package whereas lectures are concern by the supply of the labs for situations for immense categories and unmanageable teams. What is more, 
this paper provides a suggestion in implementing the software package engineering tool in cloud with descriptions in Figure two as below.

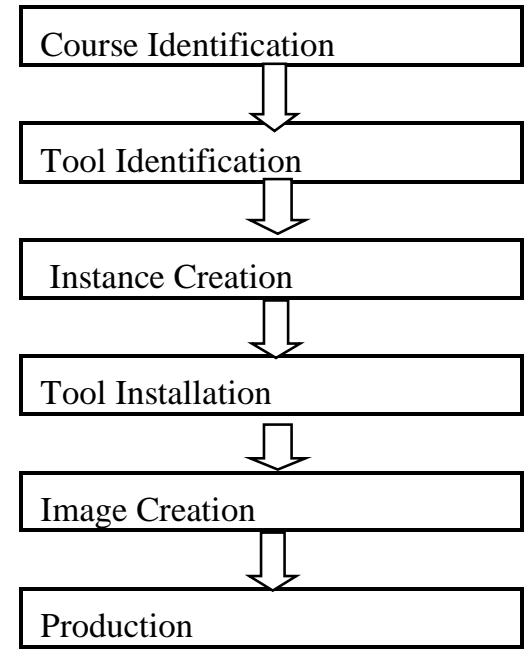

Fig. 1.2 Guidelines in implementing software engineering tools on cloud [1]

In addition, advantages of cloud computing usage are visualized through performing on multiple computers and operational systems with irrespective of time and placement.

Additionally, universities can have prime beneficiaries and this able to optimize the resources a lot of with efficiency. To conclude, cloud computing have cherish several areas weather in education or business and would have profited users through quality improvement and providing tips in implementing the tools in cloud that additionally indirectly cause benefit to the society.

\section{BIG DATA}

Now "Big Data" is magnificently proverbial for software utilize the Operational Data (OD) for software system style and maintenance activities [3]. The structure and unstructured data in operational support tools have long been rife in software system engineering field. Proposing systematic approach to the engineering field of operational info systems are fashionable analysis inside the massive data research topics [3]. Mock-ups [3] analysis study suggests satisfying growing desires for OD system in software system engineering and different fields are rising. It'll be necessary to develop basic principles and tools that enable having effective use of engineering in OD system. Mocks [3] had consistently grab best practices and use past analysis approach from different domains like databases and principles of OD system challenges. The projected feature acts as a guide to create engineering principles in OD system are 2 events that ought to have the identical context, knowledge incomplete, and data incorrect, filtered or tempered. From the options develop it's conjointly necessary to develop library basic mechanism to explain the relationships among entities for software system engineering domain [1]. The mechanisms are designed into models and used as modules by context, input missing values etc. explicit care must be taken once applying ways in OD as a result of the assumptions is also taken without any consideration and techniques might not be applicable for OD generally and for software system engineering specifically. Future add this space for analysis are implementing effective ways to spot knowledge entry drawback, clean data, augment or section events and develop sturdy ways in characteristic subject identities [4]. Whereas another analysis paper on huge knowledge describes the approaches and environments for applying clouds on huge knowledge applications [4]. Projected four areas of analytic and massive data are data management, model development and evaluation, image and user interaction conjointly business models. To conclude, huge data is seen challenge in industries to beat competitors. whereas industries able to build use of massive dat to get info then the demand of shoppers can grow, increase revenue, scale back value and increase operations. Cloud computing facilitate to elevate demand with value proportional and massive data continues to be time intense, needs pricey software system, massive infrastructures and efforts [4]; [3].

\section{ANDROID COMPUTING}

Proliferations of automaton device and application services have created demands that are applicable for software package testing techniques [5]. Previous analysis focuses on unit and user interface testing of Androids applications. Today, EvoDroid [6] is Associate in nursing biological process approach for system testing in automaton applications. EvoDroid overcomes the defect for system testing and Mahmood, et al. [6] suggests combining 2 novel techniques, automaton specific program on distinctive segments of code to be searched severally and biological process algorithmic program that offers data for such segments. though the approach [6] have shown to booming hand existing tools and techniques for automatic testing in automaton applications, it might degrade thanks to unable to consistently reason concerning input conditions. Future work exists during this are to increase the model and framework exist to produce full use of search base algorithmic program. Besides, automaton application is thought of as Event Driven software package (EDS) that's driven by many varieties of events [5]. Major issue with automaton application testing is accessing testing approaches for ancient EDS system (such as GUIs, wealthy net Applications, embedded software package, etc.) additionally accessible in automaton based mostly mobile application 
[5]. downside of automatic testing in automaton Google platform suggests techniques for fast crash testing and regression testing of the appliance [5]; [1]. The projected testing technique is aim at finding runtime crashes or visible faults on changed versions of the appliance. To conclude, automaton computing in software package engineering field of analysis is growing in testing in distinctive the proper approach and model. Proliferations of automaton device and application services have created demands that are applicable for software package testing techniques [5]. Previous analysis focuses on unit and user interface testing of Andirons applications. Today, EvoDroid [6] is Associate in nursing biological process approach for system testing in automaton applications. EvoDroid overcomes the defect for system testing and Mahmood, et al. [6] suggests combining 2 novel techniques, automaton specific program on distinctive segments of code to be searched severally and biological process algorithmic program that offers data for such segments. though the approach [6] have shown to booming hand existing tools and techniques for automatic testing in automaton applications, it might degrade thanks to unable to consistently reason concerning input conditions. Future work exists during this are to increase the model and framework exist to produce full use of search base algorithmic program. Besides, automaton application is thought of as Event Driven software package (EDS) that's driven by many varieties of events [5]. Major issue with automaton application testing is accessing testing approaches for ancientness system (such as GUIs, wealthy net Applications, embedded software package, etc.) additionally accessible in automaton based mostly mobile application [5]. downside of automatic testing in automaton Google platform suggests techniques for fast crash testing and regression testing of the appliance [5]; [1]. The projected testing technique is aim at finding runtime crashes or visible faults on changed versions of the appliance. To conclude, automaton computing in software package engineering field of analysis is growing in testing in distinctive the proper approach and model.

\section{NETWORK SECURITY}

Network security is fascinating because it permits to possess direct measure and compares the safety level provided at completely different solutions. Standard critic of past analysis is handling the rank level of vulnerabilities known that are ready to be measurable and security isn't quantitative till the difficulty is fastened [7]; [8]. Analysis on novel security metric that explicit k-zero safety known that metrics are ready to count the numerous accountable network assets compared to ranking the vulnerabilities. K-zero safety are often applied through network hardening and submits. Network hardening renders the k-zero network [8] for bigger vulnerabilities. Samples of network hardening is increasing diversity, strengthen isolation, disabling services and firewall attacks. Sub metrics applies modelling and quantifying services by fix with connected vulnerabilities. This is often a chance in selecting completely different network tough solutions [8]. The planned safety model is economical in determinant the acceptable metric for determinant the worth. Future enhancements associate degreed evaluations are required to rank the k-zero day vulnerabilities in handling inputs that are notable to vulnerabilities [8] in an application services. but analysis on network security have grownup deeper in measurement the present networks. Study on security measures regarding the one broadcast native space Network (LAN) (Ethernet [7]. A hierarchal model was planned in elucidative the intrusion detection mechanism in network security. styles of attacks are preparation section, attack section and post section. Preparation section is wherever the assailant has generic info of the network. Attack section is once the network is remotely log from another machine and accessed in another machine. Finally, post section is once the system continuous to do to try to associate degreed do changes once been hacked LAN [7] and this model is helpful for an open surroundings in real time. To conclude, network security analysis spaces in software system engineering is growing from topology area to metrics or framework extensions regards to the current technology.

\section{SOFTWARE ENGINEERING PROJECT MANAGEMENT}

Software engineering project management purpose to manage the specified set of activities and tasks consists major set of problems in coming up with of computer code comes [9] that involves computer code necessities, project coming up with that are incomplete, computer code prices and schedules that are laborious to arrange and therefore the criteria for choosing the simplest analysis, design, testing and management methodology for a computer code project that are ongoing [9]. it's quite very little that researchers grasp, the strength and weakness of a computer code kind in coming up with for a computer code engineering comes leads towards completion of your time involves setting objectives and goals, strategies, developing policies, determinative course action and creating selections [9]; [10].

additionally, obtaining the proper folks on the proper project team recommend to enhance the possibilities of success that is thru acknowledging through qualification, technical skills and skill of team members [10].

\section{CONCLUSION}

This article is helpful for analysis students in code engineering field to acknowledge the present trends of analysis topics obtainable and to manoeuvre any with the analysis gap and future works explicit within the analysis papers reviewed.

\section{REFERENCES}

[1] A. Mockus, "Engineering big data solutions," in Proceedings of the on Future of Software Engineering ACM, 2014, pp. 85-99. 
[2] Assunção, M. D., Calheiros, R. N., Bianchi, S., Netto, M. A., \& Buyya, R. (2015). Big Data computing and clouds: Trends and future directions. Journal of Parallel and Distributed Computing, 79, 3-15.

[3] D. Amalfitano, A. R. Fasolino, and P. Tramontana, "A gui crawling-based technique for android mobile application testing. In software testing, verification and validation workshops (ICSTW)," presented at the 2011 IEEE Fourth International Conference on IEEE, 2011.

[4] R. Mahmood, N. Mirzaei, and S. Malek, "EvoDroid: Segmented evolutionary testing of android apps," in Proceedings of the 22nd ACM SIGSOFT International, 2014.

[5] Review of Computer Engineering Research 2014, $\mathbf{x x x}(\mathbf{x x x}): \mathbf{x x x}$ by Gayatri Vijiyan

[6] O. Saad and M. E. Rana, "Cloud computing adoption for software engineering learning environment: Set of guidelines derived through primary research," in The Third International Conference on E-Learning and ETechnologies in Education (ICEEE2014). The Society of Digital Information and Wireless Communication, 2014, pp. 253-259. 